\title{
LOS LISPERGUER WITTEMBERG: LUCES Y SOMBRAS DE UNA SINGULAR FAMILIA ALEMANA PRESENTE EN LA HISTORIA DE ESPAÑA Y CHILE
}

\author{
THE LISPERGUER WITTEMBERG'S: LIGHTS AND \\ SHADOWS OF A SINGULAR GERMAN FAMILY \\ PRESENT IN THE HISTORY OF SPAIN AND CHILE
}

\section{DANIEL PIEDRABUENA RUIZ-TAGLE}

\section{RESUMEN}

Durante más de cuatro siglos la familia Lisperguer ha mantenido el status de ser la primera familia colonial de Chile, con grandes implicaciones en la historia, la literatura y la sociología. Descendiente de esta familia fue Catalina de los Ríos Lisperguer, apodada "La Quintrala", figura altamente mitificada, que encarna numerosos tópicos de lo que representa hoy en día la idiosincrasia chilena. Una nueva investigación ha permitido identificar a los ascendientes de los Lisperguer en Alemania, lo que, junto al descubrimiento de sus primos hispanos, los Wittemberg, ha arrojado mucha luz sobre su origen común, no exenta de algunas sombras.

Palabras clave: Lisperguer, "La Quintrala”, Wittemberg, investigación, idiosincrasia.

\section{ABSTRACT}

For over four centuries the Lisperguer family has maintained the status of being the first colonial family of Chile, with great implications in History, Literature and Sociology. A descendant of this family was Catalina de los Ríos Lisperguer, nicknamed "La Quintrala", a highly mythologized figure, who embodies many topics of what today represents the Chilean idiosyncrasy. New research has permitted us to identify "the Lisperguers' ancestors" in Germany, which together with the discovery of their His-

* Máster ( $\odot$ Universitario en Formación e Investigación Literaria y Teatral en el Contexto Europeo. Facultad de Filología. Departamento de Literatura Española y Teoría. Universidad Nacional de Educación a Distancia (UNED). Madrid, España. Correo electrónico: danielpiedrabuena@ hotmail.com 
panic cousins, the Wittemberg's, has shed much light on their common origin but not without some shadows.

Keywords: Lisperguer, “La Quintrala”, Wittemberg, research, idiosyncrasy.

Recibido: 10.10.14. Aceptado: 09.11.15.

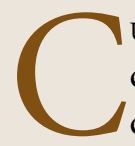

UANDO EN 1556 el conquistador Pedro Lisperguer llegaba a América, lo hacía con feudo imperial, "no embargante que es alemán y cualquier provisión que haya en contrario", siguiendo las propias palabras del César. Comenzaba así un poderoso linaje, especialmente en Chile, del que muchos historiadores han intentado desentramar su origen. Artículos como "Peter Lisperguer, ein deutscher Konquistador", de Wunder Gerd (1984), o su libro Die Familie Lisperguer in Chile (1934), o también el artículo "Don Pedro Lisperguer aus Worms", de Hans Reuss (1936) o, por supuesto, el trascendental libro Los Lisperguer y la Quintrala de Benjamín Vicuña Mackenna (1944), habían evidenciado con gran luminosidad y profusión de datos sobre el éxito de Lisperguer y su descendencia en Chile, pero apenas habían sido capaces de ofrecer un atisbo respecto del origen del conquistador en Alemania.

El gran genealogista Juan Luis de Espejo (1967, p. 526), célebre por haber sido junto a Matta Vial promotor de la Sociedad General de Historia y Geografía y uno de los fundadores de la Academia Chilena de la Historia, situaba dicho origen en el duque de Sajonia, amparándose en una certificación realizada en 1740 por don Juan Alfonso Guerra y Sandoval, rey de armas principal de Felipe $\mathrm{V}^{2}$. Se trataba de un encargo de Jorge Carlos Wittemberg, conspicuo ciudadano de Málaga, en el que se exhibían entronques reiterados entre la familia Wittemberg y la familia Lisperguer en varias de sus ramas, pero que, pese a sus ingeniosas construcciones, no justificaba de manera fidedigna sus pronunciamientos.

La república literaria de entonces, como es el caso de Eyzaguirre (1945, pp. 6-7), entre otros, asumió con suma facilidad que el apellido Wittemberg con " $\mathrm{m}$ " - repetido así treinta y nueve veces en el documento- traía su origen en la ciudad sajona de Wittenberg con " $n$ ” $y$, por tanto, no les quedaba duda de que la familia Lisperguer Wittemberg provenía de los duques de

\footnotetext{
${ }^{1}$ Biblioteca Nacional de Perú. Licencias Pedro Lisperguer, código z285, folio 576.

${ }^{2}$ Biblioteca Nacional de España, Sección Ms. No 11801, cuaderno 48, folio 1532.
} 
Sajonia en su línea Sachsen-Wittenberg. Pero esta asunción albergaba un grave problema y era que aunque en el documento sólo se mencionaban los cuarteles paternos del escudo heráldico, sus armas no guardaban ninguna relación con el ducado de Sajonia.

Luis de Roa y Ursúa, miembro también fundador de la Academia Chilena de la Historia, creía que, puesto que era un hecho histórico comprobado por los asientos de la Casa de Contratación en Sevilla que Pedro Lisperguer era hijo de Pedro Birlinguer y de Catalina Lisperguer ${ }^{3}$, el origen del conquistador alemán había que buscarlo en su ciudad natal, Worms, y no podía deducirse de certificaciones armeras. Bajo estos presupuestos recibía el 24 de abril de 1940 una carta del director de su Archivo Municipal, el Dr. Friedrich Illert, en la que le enviaba algunos datos sobre los Birling y Lisperg dispersos en varias obras de literatura, así como una lista incompleta del Consejo Comunitario de Worms donde esos nombres eran mencionados ${ }^{4}$.

Poco después, Roa y Ursúa (1945, p. 316) incorporó estas noticias a su célebre obra El Reyno de Chile, libro que estuvo avalado por el Consejo Superior de Investigaciones Científicas. Sin embargo, en ella incurrió en graves errores cronológicos, al confundir lo que pensó que eran fechas de nacimiento con lo que en realidad eran fechas de entrada en el Consejo de Worms, dando un salto de una generación, confundiendo al que creyó el padre de Pedro Lisperguer con el que en realidad era su abuelo, y al supuesto hermano con el que era su padre, trastocando por ende la identidad de todos los personajes lisperguerianos que aparecieron en su obra. Estos errores, además, los importó Espejo (1967, p. 526) a su obra.

Como solución dirimente a estos dos supuestos, Espejo (1967, p. 526) propone los datos consignados en el pasaporte de Lisperguer, en el cual aparece como hijo de Pedro Bislemberg y de Catalina Lisperguer. Conclusión insuficiente en cuanto a la relación agnaticia, dada la existencia de multitud de errores paleográficos y la carencia de otras fuentes alternativas que lo corroboren.

Un caso curioso es el de Ventura Lisperguer, capitán retirado y veedor del ejército, alemán que nunca viajó a Chile (sí lo hizo su nieto Juan Lisperguer), el cual, el 16 de diciembre de 1684, probó ante el Consejo de Indias su emparentamiento con Pedro Lisperguer y su descendencia del duque de Sajonia. Este documento encontrado por Juan Luis Espejo en el Archivo General de Indias de Sevilla fue luego publicado por Eyzaguirre (1945, pp.

\footnotetext{
${ }^{3}$ Archivo General de Indias, Contratación, 5537, L.1, folio 90v.

${ }^{4}$ Stadtarchiv Worms, Abteilung, 20 Nr 645/2 Materialsammlung Dr. Illert, 1940.
} 
6-7), pero lamentablemente se trata de un extracto que no incorpora los documentos originales que se exhibieron para acreditar tal descendencia.

Gracias a las listas publicadas por Johannes Kraus (1926a, 1926b) en la revista Der Wormsgau, hoy podemos saber que en realidad habían dos Consejos: el Dreizehner Rat y el Gemeinen Rat, que venían a ser dos cámaras de representación en el ámbito del poder municipal. Por lo tanto, Kraus, bajo el título de "Neue Quellen zur Wormser Ratsgeschichte", publicó dos listas que además contenían interesantes notas marginales: Liste der Mitglieder des Dreizehner Rates in den Jahren 1557-1609 (Lista de los miembros del Consejo de los Trece en los años 1557-1609) (1926a, pp. 89-92) y Liste der Mitglieder des Gemeinen Rats von 1440-1609 (Lista de los miembros del Consejo Comunitario de 1440-1609) (1926b, pp. 122-130). La fuente originaria en la que se basó Kraus fue un libro manuscrito encontrado en la Biblioteca Agustina del seminario de Maguncia que, junto a la temática del "Rachtung" o tratado entre el clero y los ciudadanos de Worms, ofrecía dos listas detalladas de sus consejeros.

A su vez, Schwarz (1999, p. 153) parte de la idea sobradamente constatada de que hubo dos asentamientos de la familia Birling, el primero en Worms y el segundo en Germersheim, manteniendo entronques familiares reiterados. La vinculación de Worms con Germersheim, ciudad que se encuentra un poco más al sur, resulta bastante evidente, ya que ambas ciudades estaban situadas en las riberas del Rin y por tanto conectadas por un río navegable que facilitaba todo tipo de intercambios -ya fueran humanísticos o comerciales- entre esos dos enclaves. En Germersheim la presencia de esta familia es perfectamente detectable desde la segunda mitad del siglo $\mathrm{XV}$. Sus casas y bienes raíces estaban situados principalmente en zonas suburbanas, en el Rin. La proximidad al río les permitía que pudieran vivir de los ingresos que les proporcionaba la pesca. Junto al Rin estaba también el mercado de la madera, cuyos productos eran enviados por el río desembocando en el río Kinzig o Murgtal en Württemberg, donde se cree que también se establecieron algunas ramas de la familia Birling como tratantes de madera (p. 154).

Entre los muchos miembros de la familia Birling que se establecieron en esta zona destacó especialmente Peter Birling, el padre de Pedro Lisperguer, el cual fue designado miembro del consejo municipal (Gemeinen Rat) en 1533. En las notas marginales de las listas de ese consejo se señala que ejercía la profesión de "Panni Tonsor", es decir, que era tundidor, mercader de telas, poseedor de algún tipo de industria textil y, posteriormente, fue "Holzmenger", o sea, tratante o comerciante de maderas, actividad en 
la que debió acumular una gran fortuna. Eso se demuestra con su ingreso poco después el 12 de mayo de 1541 en el Consejo de los Trece (Dreizehener Rat). Además, las notas informan que Peter Birling falleció el 24 de julio de 1567 (Kraus, 1926b, p. 125). La mucha autoridad que poseía en la ciudad de Worms se confirma como firmante, el 25 de septiembre de 1555, de la Paz de Augsburgo, donde es calificado de "Alter Stattmeister", evento al que acudió el hermano del emperador, Fernando, Rey de los Romanos (Schmaussens, 1774, p. 208).

En su artículo, Albert Schwarz (1999) menciona a varios hijos y primos de Peter Birling, sin embargo no dedica ni una sola palabra al conquistador Pedro Lisperguer. Hay que pensar que ello es normal, ya que Pedro Lisperguer se marchó de Worms con tan sólo 15 años, por lo tanto no tuvo tiempo de desempeñar ningún rol en Alemania. Lo más que podría existir es su partida de bautismo, si algún día consiguiera encontrarse. Sin embargo, hay pruebas categóricas que evidencian que Pedro Lisperguer fue hijo de Peter Birling. Esto es así ya que, como veíamos, es un hecho histórico comprobado que Pedro Lisperguer dejó constancia ante la Casa de Contratación de Sevilla, hoy Archivo General de Indias, que era oriundo de Worms e hijo de Pedro Birlinguer y de Catalina Lisperguer.

Asimismo, en la probanza que Lisperguer efectuó en Antequera el 30 de marzo de 1555, Gonzalo de Santiesteban, miembro del séquito del Conde de Feria que estuvo en Worms en la primavera y verano de 1545, declaró haber conocido al padre de Pedro Lisperguer, el cual, dijo, "era caballero principal dotado de escudos, armas y criados, uno de los trece que gobernaban la ciudad de Worms"'. Esta declaración es de grandísima importancia, ya que justamente se constata el ingreso de Peter Birling en el Dreizehener Rat el 12 de mayo de 1541, lo que cuadra a la perfección con el hecho de que el Emperador pasara por la ciudad en mayo de 1545.

No menos importantes son dos certificaciones armeras; la primera, una adición efectuada en 1650 al libro de Linajes de España de Pedro de Azcárraga, rey de armas de Navarra, donde se expresa que el padre del conquistador era cónsul de Worms en torno a esa fecha ${ }^{6}$; la segunda, a cargo del rey de armas Juan Alfonso Guerra y Sandoval, el cual aseguraba que el padre del conquistador era cónsul de Worms hacia el año $1540^{7}$.

\footnotetext{
${ }^{5}$ BNP, trámites de Pedro Lisperguer para pasar a América, código z285, folio 580.

${ }^{6}$ Azcárraga, Pedro de: Linajes, Biblioteca Nacional de España, Ms 11766, p. 366 vuelta 8.

${ }^{7}$ Biblioteca Nacional de España, Sección Ms. No 11801, cuaderno 48, fol. 1532.
} 

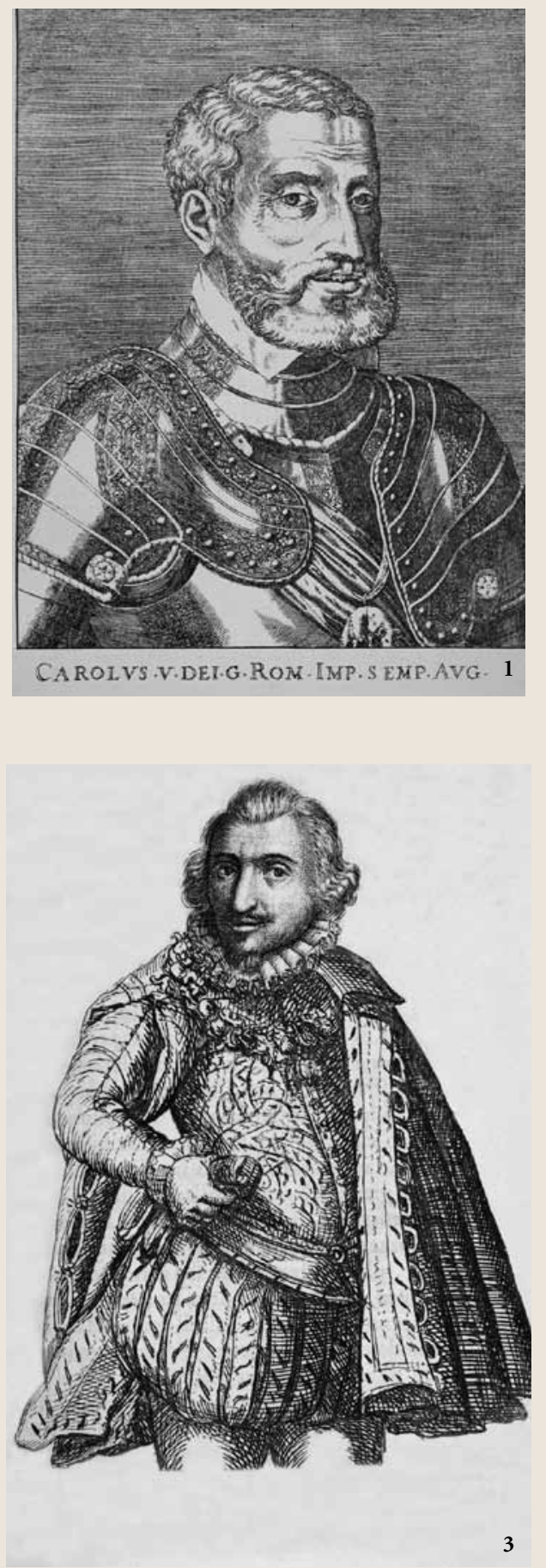

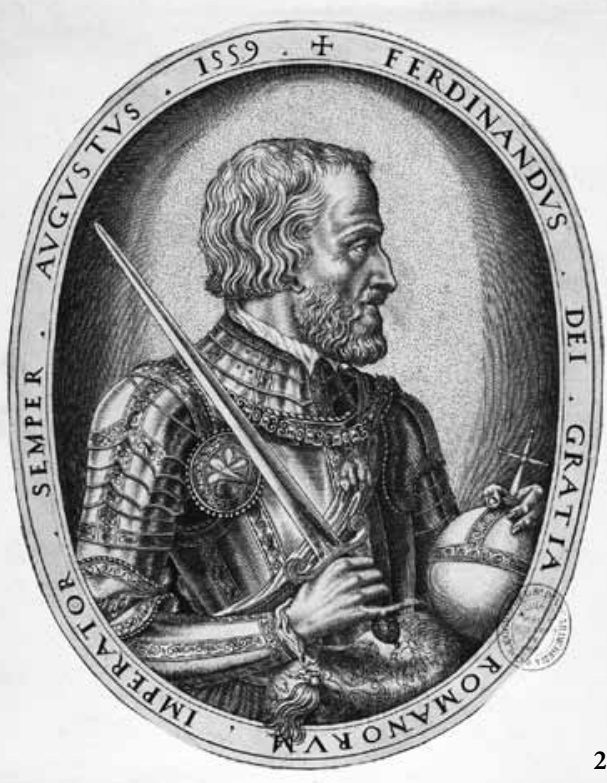

1) Emperador Carlos V. Lisperguer coincidió con el Emperador en Worms varios meses y también viajó junto a él por el sur de Alemania y los Países Bajos.

Dibujo de Heemskerck, Maarten van (1498-1574). The chief victories of the Emperor Charles the Fifth. London; and Edimburgh: Impreso privadamente para el editor [by R. Clark Edinburgh], 1870.

2) Fernando, Rey de los Romanos, hermano del emperador Carlos V. Lisperguer coincidió con Fernando en Worms.

"Retrato de Fernando I, Emperador de Alemania" (aguafuerte y buril; 181 x $144 \mathrm{~mm}$ ). Autor: Heyden, Pieter van der (ca. 1530-ca. 1572). Amberes: Cock excv., 1559. Iconografía Hispana, 3163-1.

3) Don Gómez Suárez de Figueroa, V conde de Feria. Lisperguer se marchó de Worms en 1545 junto al IV conde de Feria, don Pedro Fernández de Córdoba. Después se separó del Emperador en Utrecht y continuó con el Conde hasta Andalucía. Tras la muerte de don Pedro en Priego en 1552, Lisperguer continuó en la casa de Feria con el nuevo conde, don Gómez Suárez de Figueroa, embajador de Felipe II en Inglaterra y miembro de su Consejo de Estado. Con éste viajó Lisperguer a Londres, donde permaneció junto al monarca hasta finales de 1554, tras lo que volvió con Alderete y el virrey Hurtado de Mendoza a España. En España estuvo cerca de un año realizando diversos trámites administrativos antes de viajar a América.

Historia o vero Vita di Elisabetta, Regina d'Inghilterra. Buril, $137 \mathrm{x}$ $86 \mathrm{~mm}$. Amsterdamo: Apresso Abramo Wolfgang, 1693. Iconografía Hispana, 2946-72 (Felipe II). 
En adición a lo anterior tenemos el testimonio de Schwarz (1999, p. 155), quien, en su artículo sobre los Birling, asegura que el cónyuge de Peter Birling (o Birlinguer) procedía de la familia concejil de los Lisberg (o Lisperg como es indistintamente mencionado), lo que implica la identificación de los padres del conquistador en la literatura alemana. También tenemos el propio testimonio del Dr. Illert, el cual, en su contestación a Roa y Ursúa y en referencia a Peter Lisperg, dijo: "Se puede dar por seguro el parentesco con la influyente familia Lissberg de Worms" . Por si fuera poco, Pedro Lisperguer portó el nombre de Pedro como su padre (Peter). Pero además él mismo en Chile puso a sus propios hijos los nombres de Juan Rudolfo, Pedro, Catalina. Estos nombres hacen una clara alusión a su abuelo Johann (Hans), a su bisabuelo Rudolph, y a su madre Catharina Lisperg.

Dicho lo anterior, se constata que la entrada de la familia Lisperguer en el Gemeinen Rat se produce según el siguiente orden: 1474, Hamman Liesberg; 1504, Hans Lisperg; 1511, Rudolf Liesperg (Kraus, 1926b, p. 122). Tanto Hans como Rudolf se matricularon en la Universidad de Heidelberg, una de las más antiguas, prestigiosas e importantes de Alemania; el primero, el 9 de mayo de 1493, y el segundo lo hizo antes, el 5 de noviembre de 1464 (Toepke, I, 1884, pp. 312 y 405), por lo que, frente a lo que sostiene Roa y Ursúa (1945, p. 316), en términos cronológicos es mucho más probable que Hans fuera el abuelo de Pedro Lisperguer y no Rudolf.

Por otra parte, Karl Heinz Armknecht (1951) asegura que la familia Birling, comerciantes de madera, tienen su origen en Hans Birling, miembro del Gemeinen Rat en 1468, del cual proceden seis generaciones de esta importante familia (151). Así, pues, está constatado en las entradas del Gemeinen Rat que a este órgano se incorporaron sucesivamente: 1468, Hans Birling; 1475, Jacob Birling; 1503, Peter Birling (también en 1513, según Schwarz); 1533, Peter Birling, primero "pani tonsor" y luego tratante de maderas, fallecido el 24 de julio de 1567; 1570, Hans Birling, tratante de maderas, "Petri filius", es decir, hijo de Peter Birling, muerto a la edad de 57 años, el 29 de diciembre de 1597 y finalmente en 1588 Steffan (o Stephan) Birling, procedente de Gemersheim, tratante de maderas (Kraus, 1926b, pp. 122-129).

Respecto al Consejo de los Trece, las listas y sus preciosas notas marginales recogen que Peter Birling (padre de Pedro Lisperguer) entró en el Consejo el 12 de mayo de 1541 y que falleció el 24 de julio de 1567. El 18 de abril de 1588 entra en la augusta corporación Hans Birling, "Petri filius"

\footnotetext{
${ }^{8}$ Ibídem, Stadtarchiv Worms, Abteilung, 20 Nr 645/2.
} 
(hijo de Peter Birling), en lugar de Hans Kegels y muere el 29 de diciembre de 1597, "aetatis anno 57, 2 M.D. masculus suae familiae ultimus". Es decir, Hans Birling, hermano de Pedro Lisperguer, murió a la edad de 57 años y 2 meses, siendo el último varón de su familia. El 2 de febrero de 1598 le sucede Stephan Birling, procedente de Germersheim, por un estrecho parentesco que le unía (Kraus, 1926a, pp. 89-92), siendo seguramente su primo.

Tras la muerte de Hans Birling en 1597 los intereses de la familia en el Consejo de Worms pudieron ser preservados a través de Stephan Birling. Fue éste un hombre preeminente y sabio, con gran influencia tanto en Worms como en Germersheim. Comienza su carrera en 1568, momento en que fue designado por el Elector Federico III como alcalde o presidente del Consejo (Schultheissen) y en el mismo año era designado como agente de aduanas en la zona del Rin (Rheinzoller). En tal cargo ostentaba la más alta magistratura, controlando junto al escribano de la aduana, los ingresos o recaudación de la aduana del Rin. Alrededor de 1575 se trasladó a Worms, ingresando posteriormente en 1588 en el Consejo Comunitario (Gemeinen Rat), siendo calificado de "Holtzmenger", es decir, tratante o comerciante de maderas. El 2 de febrero de 1598 ascendió al Consejo de los Trece en lugar de Hans Birling, lo que suponía un importante avance en su carrera.

Stephan Birling murió en 1618 siendo burgomaestre y maestro de obras (Baumeister), según rezaba el título de "responsable de construcciones" (Bauwesen), que portaba como miembro del Consejo. Antes que él murieron sus dos esposas: María Pöttiger, fallecida el 1 de noviembre de 1596, y Ana Rosenstil, fallecida en 1605. Después de haberse casado dos veces, ordenó en 1601 para sí y sus dos mujeres la construcción de un sepulcro, que debía estar situado en el Cementerio Luterano, en el cual, junto a las armas de alianza Birling-Rosenstil y las armas de los cuatro antepasados, se colocaba una larga inscripción en latín. Stephan tuvo con su primera mujer, María Pöttiger, dos hijos de los que no han trascendido más noticias. De su segundo matrimonio con Anna Rosenstil tuvo una hija llamada Anna María, la cual se casó con Philipp Schmitz. Todo sugiere que Stephan se casó una tercera vez con una tal Helena, que es mencionada en 1605 y 1611.

Schmitz procedía de una familia de funcionarios de Pfälz. Su padre fue alcalde o presidente del Consejo (Schultheiss) en la ciudad de Leiningischwesterburgischen Grünstadt. Igual que su padre, él también se comprometió con la política del Estado: en 1604 perteneció al "Vierern" y en 1606 al Gemeinen Rat de Worms. En sus listas se puede leer como éste era "yerno de Stephan Birling" y además "Grunstadiensis", es decir, procedente de Grünstadt. En el momento de la muerte de Schmitz en 1617 tenía su mujer un sepulcro construido, que antiguamente se encontraba en el Cementerio 
Luterano y que hoy se halla en el museo de la ciudad. La inscripción recuerda también su muerte, así como la de su hija conjunta, Anna Katharina, cuya suerte es desconocida (Schwarz, 1999, p. 155).

En cuanto a las hermanas de Pedro Lisperguer y Hans Birling, Schwarz menciona en primer lugar a Verónica, la cual se casó con el presidente del consejo de Worms (Schultheissen) y consejero Bernhard Schlatt, el cual, al igual que su suegro, se dedicó inicialmente al comercio textil y luego al negocio de la madera (p. 155). Según Armknecht (1951), el iniciador de esta familia de farmacéuticos o boticarios fue Christoph Schlatt, el cual formó parte en 1521 del Gemeinen Rat y como él otros cinco miembros de esta familia formaron parte del ilustre consejo (p. 406).

Christoph Schlatt, consejero en 1521 y dedicado a la "Pharmacopola", tuvo un hermano llamado Matthías Schlatt, dedicado a la misma actividad, según atestiguan las notas marginales de las listas del consejo, el cual entró como su hermano en 1555 en el Gemeinen Rat y murió el 17 de julio de 1570. Asimismo consta que tanto Matthías como Bernhard se matricularon en 1531 en la Universidad de Marburg (Falkenheiner, 1904, p. 144). Igualmente se verifica como el mismo Matthías Schlatt, procedente de Worms, se matriculó poco después en la Universidad de Heidelberg, el 20 de abril de 1533 (Toepke, 1884, p. 154). Este hecho de pertenecer a varias universidades o "peregrinatio academica" era un fenómeno totalmente habitual en la época.

Bernhard Schlatt, hijo de Christoph, marido de Veronica Birling y cuñado de Pedro Lisperguer, entró en el Gemeinen Rat en 1563, constando que fue primero "Leinenkremer", tratante de telas y luego, "Holtzmenger", comerciante de maderas. Posteriormente, el 11 de marzo de 1573 entró en el Consejo de los Trece, en lugar de Haug Peuschels, constando en sus notas marginales que fue "gener Petri Birling", es decir, yerno de Peter Birling. Bernhard murió el 19 de septiembre de 1592 a la edad de 52 años y su mujer, cuatro años más tarde, el 17 de julio de 1596. Un fragmento de su sepulcro, que una vez estuvo en el Cementerio Luterano, hoy se encuentra en el Museo de Worms.

En el fragmento, junto a sus padres, se encuentran de rodillas en postura de oración ocho niños: seis hijos, una niña llamada Margretha y un niño envuelto en paños (Schwarz, 1999, p. 155), todos ellos sobrinos de Lisperguer. A su vez, la representación está flanqueada por los escudos de armas de la Casa: cuatro armas y dos inscripciones. Entre las armas se reconocen las de Meiel (o Mejel), Lisberg y Marchand, lo que indica un estrecho parentesco con estas destacadas familias de consejeros de Worms (Füchs, 1991, p. 395). 


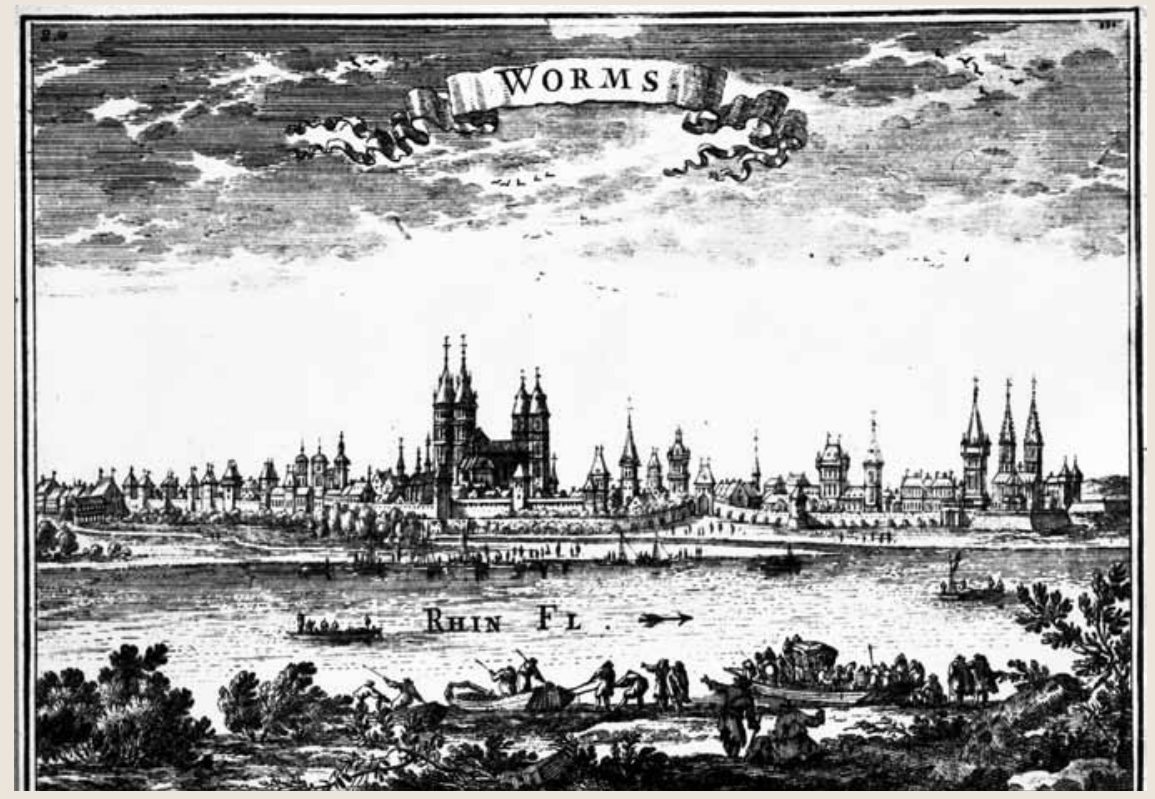

Stadtarchiv Worms. Abt. 217 Kasten 3 Nr. 17, um 1690 (Reproduktion M07341).

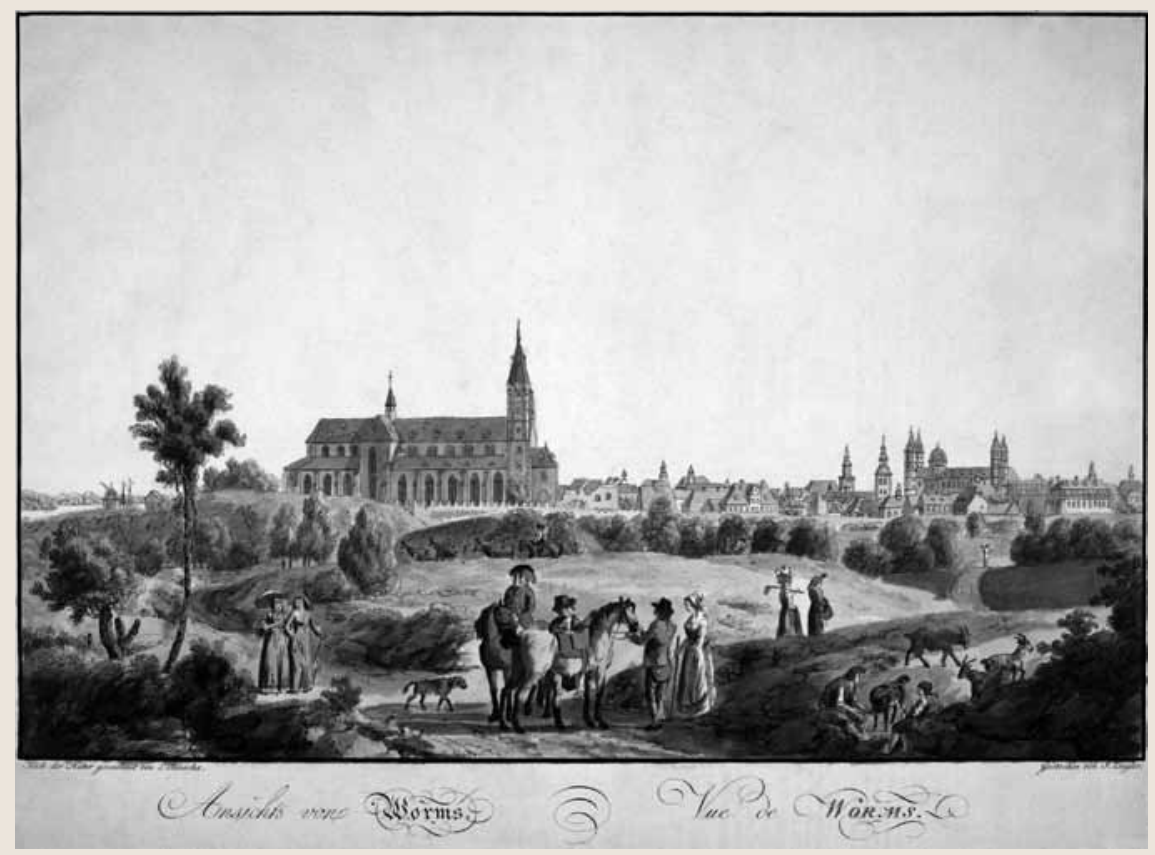

Stadtarchiv Worms. Abt. 217 Lade 6 Nr. 31c, 1780. 
De los sobrinos varones de Lisperguer (hijos de Bernhard y Verónica) se conocen varios de sus nombres. El más importante sería Bernhard Christoph Schlatt, educado en la Universidad de Heidelberg, donde consta que Bernhardus Christophorus Schlat, Wormatiensis, se matriculó el 23 de mayo de 1581 (Toepke, 1889, p. 95). En el año 1590 se incorporó en el Gemeinen Rat, figurando que fue "Friescheimer". El 9 de octubre de 1592 ingresó en el Consejo de los Trece en lugar de su padre (Kraus, 1926a, p. 92). Se casó con Anna María Weber, hija del prestigioso doctor jurista Weber de Worms. Otras fuentes señalan que Bernhard Christoph fue consejero en 1600 y Schultheiss o presidente del Consejo de Worms en 1607 (Körner, 1971, p. 407). No obstante que su carrera era prometedora, murió el 6 de mayo de 1608, "vespere anno aetatis 44 ipsa hora mortis complete quae fuit octava" (Kraus, 1926a, p. 92).

Tras su muerte, su viuda se casó en Worms el 22 de febrero de 1611 con Johan Steffan von Cronstetten, nacido en Frankfurt el 16 de agosto de 1575, ocupando en Worms los cargos de consejero en 1605, Stättmeister y luego Ratssenior, muriendo en dicha ciudad el 16 de agosto de 1624, estando enterrado en la Iglesia de St. Magnus. Tras la muerte de éste, Anna concierta un tercer matrimonio en Worms el 19 de septiembre de 1636 con Michael Stromer, que en 1609 ingresó en el Gemeinen Rat, y luego fue miembro del Consejo de los Trece en 1618 y 1621, Shultheiss en 1627 y Stättmeister de Worms en 1631, muriendo el 21 de julio de 1637 (Körner, 1971, p. 405).

Hermano de Bernhard Christoph fue Andreas Schlatt, el cual en 1591 ingresó en el Gemeinen Rat, teniendo noticias también de su primo, Johann Philippus Schlatt, hijo de Mattías, que asimismo se incorporó al Gemeinen Rat en 1599, donde figura en notas marginales que su profesión fue la de "pharmacopola" y que falleció el 31 de enero de 1607 a la edad de 58 años (Kraus, 1926b, p. 129). Por otra parte, como se ha podido observar la familia Birling también se vinculó con la destacada familia Weber. Tenemos la evidencia ya referida del matrimonio Bernhard Christoph con Anna Weber, así como la de Barbara Birling, probablemente hermana o prima de Hans Birling, que fue mujer de Michael Weber, matrimonio del que se conoce un hijo, Johann Caspar Weber' ${ }^{9}$ y la presencia de otro miembro de esta familia, Baltasar Weber, de Worms ${ }^{10}$, probablemente hermano del anterior. Así, pues, el iniciador de este linaje en Worms fue Stephan Weber, el

${ }^{9}$ Hessisches Hauptstaatsarchiv Wiesbaden (HHSTAW), Reichskammergericht, 2047.

${ }^{10}$ HHSTAW, Fürstentum Nassau-Oranien: Akten (Altes Dillenburger Archiv) (171): C 117. 
cual entra en el Gemeinen Rat en 1550 y muere el 2 de diciembre de 1569. En cuanto a su profesión las listas dicen que fue "primo müller in der Stifftmühl zu Neuhaufen, postea Becker zum Affen"; es decir, molinero en Neuhaufen y hornero o panadero en Affen (en las anotaciones marginales portadas por sus descendientes aparece designado como notario). Posteriormente, entró en el Consejo de los Trece el 19 de abril de 1560, en lugar de Marx Dudenhofers. Hijos de Stephan fueron: Michael y Philips Stephan Weber. Respecto al segundo, Philips Stephan, hijo del notario Stephan, magistrado, entra en el Gemeinen Rat en 1570 y falleció el 4 de mayo de 1576. Este podría ser el padre de Anna Weber, mujer de Bernhard Schlatt. Hijo de Philips Stephan fue Philippus o Johan Philippus (en latín), notario, nieto de Stephan, ingresó en el Gemeinen Rat en 1593 y posteriormente en el Consejo de los Trece el 29 de julio de 1601 en lugar de Hans Caspar Mejels, donde es calificado de "hommo ambitiosissimus"(Kraus, 1926a, p. 92).

Igualmente, fue hijo de Stephan, iniciador del linaje en Worms, Michael Weber, casado con Barbara Birling, el cual se incorporó al Gemeinen Rat en 1566 y murió a la edad de 55 años el 5 de octubre de 1598, figurando como su profesión la de "Pistor en Stengen", o sea hornero o panadero. A continuación ingresó en el Dreizehner Rat, el 19 de mayo de 1573 en lugar de Hans Frisch, en cuyas listas figura que murió el 6 de octubre de 1598 a la edad de 55 años, 7 meses y 16 días. Sus hijos tuvieron brillante carrera. Entre ellos, Johan Christoph Weber, educado en la Universidad de Marburg, en la que se matriculó en 1593 (Falkenheiner, 1904, p. 171) y posteriormente en la de Heidelberg, donde figura su procedencia, "Wormatiensis" y su fecha de ingreso el 21 de enero de 1595 (Toepke, 1889, p. 177).

En 1598 entra en el Gemeinen Rat, donde figura como "studiosus iuris" y el 12 de agosto de 1603 ingresa en el Consejo de los Trece "in locum S. Rauschij, admodum iuvenis" (Kraus, 1926b, p. 129). En 1633 junto a su madre Barbara Birling, como viuda de Michael Weber, encabeza una demanda contra Ludwig Emich conde de zu Leiningen-Westerburg, Oberbronn, por una pensión pendiente de 40 florines al año, según una deuda que había contraído en 1587 el abuelo de éste, Philipp I. Conde de Leiningen-Westerburg, de 800 florines, con el difunto Michael Weber ${ }^{11}$.

Hermanos de Johan Christoph Weber fueron Johan Georg, el cual entró en 1605 en el Gemeinen Rat, donde consta que fue hijo de Michael, y de profesión "Lignarius", o sea carpintero, y Johan Caspar Weber, que también ingresó en el mismo consejo en 1606, donde figura que fue hijo de Michael.

${ }^{11}$ HHSTAW, Reichskammergericht, 2047 Barbara Birling, Witwe Michael Weber, Worms. 
Otras fuentes señalan que por aquel tiempo existió un Baltasar Weber, procedente de Worms, probablemente hermano de los anteriores o cuanto menos perteneciente a este clan, el cual, según un escrito de 1593 efectuado en Nassau-Diez, estaba incurso en una reclamación judicial como descendiente propietario de Hans Birling, de Worms ${ }^{12}$.

Su lápida y armas se encuentran en el Museo de Worms, donde aparece como licenciado y luterano, fallecido en 1614 (Schrecker, 2007, p. 77). Igualmente consta como otro Baltasar Weber, procedente de Worms, estudió en la Universidad de Heidelberg, donde se matriculó el 8 de mayo de 1615 (Toepke, 1889, p. 273). Asimismo, figura que Georgius Balthasar Weber, Wormatiensis, estudió Teología en la Universidad de Estrasburgo, donde se matriculó el 27 de mayo de 1653 (Knod, 1902, p. 336).

Otra hermana de Pedro Lisperguer, cuyo nombre aún se desconoce, se sabe que se casó con Jacob Schaaf, el cual entró en el Gemeinen Rat en 1572, donde figura como "Profectus Heidelbergam", es decir, que ostentó algún tipo de magistratura o cargo gubernativo en Heildelberg, figurando en las listas del consejo como "Wullenkremer" o tratante de lanas, y además "gener Petri Birling", o sea yerno de Peter Birling. Fue Jacob hijo de Eberhartt Schaaf, el cual ingresó en el Gemeinen Rat en 1534, y fue "sepultus ad S. Lampertum" el 10 de mayo de 1566. Igual que su hijo está registrado que fue "Wollenkremer tandem: antea enim fuit hospes im Kaufthaus". Además perteneció al Consejo de los Trece en el que entró el 31 de diciembre de 1544 y donde está registrado igualmente que falleció el 10 de mayo de 1566. Es muy probable que padre de Eberhartt y abuelo de Jacob, haya sido Wilhelm Schäfer, el cual se unió al Gemeinen Rat en 1498 y murió en 1534 (Kraus, 1926b, p. 127; Kraus, 1926a, p. 90).

Por último, añade Schwarz (1999, p. 155) en su artículo, que Verónica Birling tuvo otra hermana que se enlazó a un miembro de una familia que se llamaba Marchand, Marckchart o Marckort, cuya descendencia masculina es detectable en el Gemeinen Rat desde la segunda mitad del siglo XV. Su hijo Johann Christoph Marchard sería elegido en 1612 para el consejo municipal. En la matrícula de la Universidad de Marburg está constatado que en sus ilustres aposentadurías cursó estudios en 1596 Johan Christoph Marckardt (Falkenheiner, 1904, p. 103), como está igualmente evidenciado que Johannes Christoph Marckhard, procedente de Worms, se matriculó en la Universidad de Heidelberg el 22 de julio de 1599 (Toepke, 1889, p. 198) y antes que él un Christoph Marquarth, también procedente de

\footnotetext{
${ }^{12}$ HHSTAW, Fürstentum Nassau-Oranien: Akten (Altes Dillenburger Archiv) (171): C 1173.
} 
Worms, lo había hecho el 10 de diciembre de 1553 (Toepke, 1884, p. 556). En 1617 murió como el último de su linaje. Su sepulcro se encuentra en la Iglesia Magnuskirche de Worms (Schwarz, 1999, p. 155). En él sus armas e inscripciones se corresponden con las de: Marchard (Marchardt), Kraft, Birling y Lisperg (Füchs 1991, p. 463), lo que refuerza el parentesco con los padres de Pedro Lisperguer. Tanto de las listas del Consejo como por el artículo del Sr. Armknecht (1961, p. 122) se evidencia que Johan Christoph tuvo un hermano llamado Hans Caspar Marchart o Marchard, el cual fue también miembro del Gemeinen Rat en 1608, siendo ambos hijos del doctor Caspar Marchard.

Volviendo a las certificaciones armeras, interesante por su novedad es un expediente de nobleza de la familia Wittemberg, recopilado en un nobiliario de Pedro de Azcárraga, en el que el rey de armas Juan de Mendoza hizo unas adiciones en el siglo XVII. Aquí nuevamente se menciona a Pedro Lisperguer como perteneciente a este clan, describiendo las armas paternas y maternas de su escudo heráldico, lo que ha sido fundamental para establecer el origen de la familia ${ }^{13}$. Provechosa, si bien no determinante, es una "información ad perpetuam", dada a Juan Wittemberg Dreyers, el fundador del linaje en Andalucía y realizada el 13 de febrero de 1699, refrendada y sellada por los procónsules y senadores de la ciudad de Hamburgo ${ }^{14}$. En iguales términos se encuentran unas "pruebas de catolicidad", practicadas a favor de Juan Wittemberg Arizón, futuro deán de la Catedral de Málaga y promovidas ante los padres jesuitas de Hamburgo el 16 de abril de 1730, refrendadas por Antonio Casado y Velasco, marqués de Monteleón y embajador de Felipe $\mathrm{V}$ en la misma ciudad ${ }^{15}$.

Muy interesante es una Provisión de Nobleza (equivalente a una Real Ejecutoria de Hidalguía) ganada por los hermanos Jorge Carlos y Joseph Wittemberg Aguilar el 31 de marzo de 1745 ante la Real Chancillería de Granada, donde los hermanos Wittemberg aseguraron descender de la casa Wittemberg en Alemania La Baja ${ }^{16}$. A su vez esta misma Provisión fue ex-

${ }^{13}$ Azcárraga: "Recopilación General de Linajes de España”. BNE, Ms 11766, p. 366.

${ }^{14}$ Archivo Histórico Catedralicio de Málaga. Legajo no 48, pieza nº41, año 1730 . Archivo de la Real Chancillería de Granada. Concesión de Real Provisión Ordinaria el 31 de marzo de 1745 a don Jorge y don José Wittemberg, vecinos de Málaga, litigantes que disputan con el Concejo de Benamocarra. Caja 14426, pieza 42.

${ }^{15}$ Archivo Histórico Catedralicio de Málaga. Legajo no 48, pieza no 41, año 1730. Archivo de la Real Chancillería de Granada. Concesión de Real Provisión Ordinaria el 31 de marzo de 1745 a don Jorge y don José Wittemberg, vecinos de Málaga, litigantes que disputan con el Concejo de Benamocarra. Caja 14426, pieza 42.

${ }^{16}$ Ibídem. 
hibida con gran esplendor en un instrumento realizado en 1776 a favor de dos nietos de Jorge Carlos Wittemberg ante las autoridades de la Marina, donde varios testigos de la mayor reputación y nombradía declararon bajo juramento que:

los Witemberg descienden de los Duques de Wittemberg, Mecxin y Mumpelgard y de los Condes de Aurach y Tux en el reino de Alemania de cuyo soberano conservan un privilegio muy honroso que da una cabal idea del lustre de esta familia que ha visto el testigo por cuya causa han tenido a bien enlazar con ella varios títulos de Castilla ${ }^{17}$.

Estos ducados y condados se corresponden en la actualidad con Württemberg (también denominado Wirttemberg, o Wittemberg) Alemania; Metzingen, Alemania (próximo a Suiza); Mömpelgard (ahora Montbeliard), Francia; Urach, Alemania; Tux, Austria, señoríos todos ellos que concuerdan (con la salvedad de Tux) con las posesiones tradicionales de los duques de Württemberg y que coinciden con las representaciones heráldicas portadas por la familia Wittemberg en España, con la sola excepción de la cimera. Esto ha abierto una brecha en la creencia de la descendencia de la familia Lisperguer Wittemberg de los duques de Sajonia.

Sin embargo, tras la comprobación minuciosa de la genealogía de los duques de Sajonia y Württemberg, no ha sido posible encontrar ningún vínculo que permita eslabonar a la familia con su solar primitivo. El doctor Ludwig, miembro del Sächsisches Hauptstaatsarchiv Dresden ${ }^{18}$, demuestra con datos sólidos que los duques de Sajonia-Wittenberg no produjeron más descendencia masculina a partir de 1422. En su opinión, si la familia Lisperguer Wittemberg procede de una línea ilegítima jamás será tratado por la literatura científica. Todo ello le lleva a pensar que se trata de una leyenda de la familia, promovida por ella misma para aumentar su estatus social. Este mismo argumento se puede trasladar a la descendencia de esta familia alemana respecto a los duques de Württemberg.

Otros especialistas como Taboada (1991, p. 135), Barros Arana (1830, III, p. 297, nota 496), Thayer Ojeda (1939, p. 211) nos hablan de entroncamientos fantásticos, de vanidad nobiliaria, de la falta de credibilidad de unas informaciones que no acreditan la fehaciencia de los datos que exponen. Luego está la disquisición nada baldía de si en el caso de Wittemberg

\footnotetext{
${ }^{17}$ Real Archivo de la Marina, Madrid. Expediente no 1515, p. 32, año 1776.

${ }^{18}$ Carta del Dr. Jörg Ludwig, miembro del Sächsisches Hauptstaatsarchiv Dresden, a Daniel Piedrabuena Ruiz-Tagle. Dresde, 30 de marzo 1998, referencia 7512.2/510.98.
} 
nos encontramos ante un nombre gentilicio o ante un toponímico o nombre de procedencia. Sin embargo, habiendo ganado la familia un pleito de suma importancia, que le permitió enlazar con varios títulos de Castilla tanto en Chile como en España, siempre habrá un campo de especulación para divisar un reguero de luz entre las sombras.

\section{REFERENCIAS}

Armknecht, K. H. (1951). “Wormser Familienwappen, Ratherren”. Der Wormsgau, Zeitchrift der Kulturinstitute der Stadt Worms und des alterumsvereins Worms. Dritter Band, 7 Hefte, p. 151.

Barros Arana, D. (1830-1907). Historia General de Chile, 2a edición, Tomo III, Capítulo 20, Santiago de Chile, Editorial Universitaria, Centro de Investigaciones Diego Barros Arana.

Espejo, J. L. (1967). Nobiliario de la Capitanía General de Chile. Santiago de Chile: Editorial Andrés Bello.

Eyzaguirre, J. (1945). "La Quintrala en su lucha con la Iglesia". Boletín de la Academia Chilena de la Historia, 32.

Falkenheiner, W. (1904). Personen und ortsregister zu der Matrikel an der Annalen der Universität Marburg, 1527-1562. Marburg, Alemania: Elwert.

Füchs, R. (1991). Die Inschriften der Stadt Worms. Wiesbaden, Alemania: Reichert.

Knod, G. C. (1902). Die Alten Matrikeln der Universität Strassburg, 1621 bis 1793 Personen - und Ortsregister. Strasburg, Francia: Karl J. Trübner.

Körner, H. (1971). Frankfurter Patrizier. Historisch-Genealogisches Handbuch der Adeligen Ganerbschaft des Hauses Alten-Limpurg zu Frankfurt am Main. Münich, Alemania: Ernst Vögel.

Kraus, J. (1926a). "Neue Quellen zur Wormser Ratsgeschichte, I- Liste der Mitglieder des Dreizehner Rates in den Jahren 1557-1609". Der Wormsgau 1, $33,89-92$.

Kraus, J. (1926b). "Neue Quellen zur Wormser Ratgeschichte, II- Liste der Mitglieder des Gemeinen Rats von 1440-1609”. Der Worsgau 1, 33, 122-130.

Reuss, H. (1936). "Don Pedro Lisperguer aus Worms". Volk und Scholle, 145148.

Roa y Ursúa, Luis de (1945). El Reyno de Chile 1535-1810: estudio histórico, genealógico y biográfico. Valladolid, España: Tall. Tip. Cuesta.

Schmaussens, J. J. (1774). Corpvs vris Pvblici S.R. Imperii Academicvm. Leipzig, Alemania: Gleditschens Buchhandlungs.

Schrecker, T. (2007). "Familiennamen auf Grabsteinen in Museum der Stadt Worms". Andreasstift. Pfälzisch-rheinisch Familienkunde, 16-56.

Schwarz, A. (1999). "Die Wormser Familie Birling”. Pfälzisch-Rheinische Familiekunde, XLVIII Jahrgang, Band 14, Heft 4, 153-157. 
Taboada Roca, M. Conde de Borrajeiros (1991) Las probanzas de hidalguía antes y después de 1836. Madrid, España: Hidalguía.

Thayer Ojeda, T. (1939-1943). Formación de la sociedad chilena, vol. III, fol. 211, Santiago, Chile: Prensas de la Universidad de Chile.

Toepke, G. (1884). Die Matrikel der Universität Heidelberg, I, 1386 bis 1553. Heidelberg, Alemania: Nendeln/Liechtenstein : Kraus.

Toepke, G. (1889). Die Matrikel der Universität Heidelberg, II, 1554-1662. Heidelberg, Alemania: Nendeln/Liechtenstein: Kraus.

Vicuña Mackenna, B. (1944). Los Lisperguer y la Quintrala. Edición crítica de Jaime Eyzaguirre. Santiago, Chile: Zig-Zag.

Wunder, G. (1934). Die Familie Lisperguer in Chile. Santiago, Chile: Der Deutschen Wissenschftlichen Vereins zu Santiago, N.F.Z.

Wunder, G. (1984). "Peter Lisperger, ein deutscher Konquistador”. In Bayer, Bürger, Edelmann (pp. 373-379). Sigmaringen, Alemania: Thorbecke. 